\title{
Effects of Vibrotactile Feedback on Human Control Performance in a Dynamical Stabilization Task
}

\author{
Hendrik Börner ${ }^{1}$, Satoshi Endo ${ }^{1}$, Antonio Frisoli ${ }^{2}$, Sandra Hirche ${ }^{1}$
}

\begin{abstract}
While research has demonstrated how vibrotactile devices can be effectively used to guide human behavior, efficient mappings of vibration patterns for spatial guidance in time-critical dynamical tasks have not yet been understood. In this paper, we contrast two types of action-dependent, haptic stimulus designs to demonstrate the different effects of vibrotactile feedback on the human control performance. A wireless bracelet is used to provide patterns of vibrotactile stimuli in real-time, representing either optimal hand velocity or acceleration for the stabilization of an inverted pendulum. The optimal control behavior is supplied by a linear quadratic regulator. The analyses of the participants' stabilization and learning behavior revealed a significant improvement caused by the additional velocity-dependent feedback. The results are consistent with previous research, which indicates that the human sensory-motor system is generally more sensitive to velocity than acceleration information. In summary, the present paper suggests how human-centric vibrotactile stimuli should be designed and how they can be effectively transmitted to the human user for time-critical behavioral guidance.
\end{abstract}

\section{INTRODUCTION}

The human brain processes sensory information available in the environment when planning and executing behavior. However, due to uncertainties arising from noise in sensory and motor processes or in the environment, the resulting behavior may not be desirable for successfully completing a given task. In order to reduce task-related uncertainty and guide behavior in a desired direction, several haptic interfaces have been developed - such as for spatial navigation [1], [2] or as part of motor training [3], [4]. In particular, the use of vibrotactile feedback has attracted much attention for its low-cost, readily wearable and non-invasive features [5]. Although its effectiveness has partially been questioned, previous studies have demonstrated improvement of user performance by vibrotactile feedback - especially when other sensory modalities are unavailable or overloaded [6].

While these studies have reported how vibrotactile stimuli can be designed to maximize information transmission rate or subjective intuitiveness [3], the efficiency of information processing as part of human sensory-motor coordination has not been sufficiently explored. Neural processing efficiency becomes a critical issue, when a source of feedback is degraded, or the motor task is inherently unstable, as it requires temporally stringent sensory-motor coordination.

In a previous study, vibrotactile feedback based on optimal control behavior of a linear quadratic regulator (LQR) was

\footnotetext{
${ }^{1}$ Chair of Information-oriented Control, Faculty of Electrical Engineering and Information Technology, Technische Universität München, D-80333 Munich, Germany. \{hendrik. boerner, s. endo, hirche\}@tum.de

${ }^{2}$ PERCRO Laboratory, Scuola Superiore Sant'Anna, 56127 Pisa, Italy. a.frisoli@sssup.it
}

used to guide a human user in stabilization of an inverted pendulum [7]. A vision-based motion tracking system was used and the vibrotactile feedback represented the LQR reference acceleration-profile. Since proprioceptive sensors as well as the internal models of limb dynamics are more susceptible to the velocity profile of the arm dynamics than its acceleration profile [8], the former feedback design may result in more pronounced performance improvement. Therefore, in this paper, we contrast two action-dependent, LQR-induced vibrotactile feedbacks - velocity-profile and acceleration-profile based - for effectiveness in guidance of human control behavior in a time-critical dynamical task.

We designed an experiment based on the stabilization of a virtual inverted pendulum, in which a wireless bracelet emits vibrotactile feedback on the wrist of the human user. In our study, discrete vibration patterns were used, so as to avoid the rapid adaptation of the Pacinian corpuscles to continuous stimulation [9]. Instead of tracking the user's hand movement, we kinaesthetically rendered the movement of the virtual cart. In addition to serving as an input device, the resulting interface supplies force feedback, essentially closing the control loop. In order to evaluate the extent to which vibrotactile feedback can support human control performance with reduced visual feedback quality, the effects of the vibrotactile stimulus designs in combination with partially degraded visual feedback were also studied. The obtained results were statistically analyzed with respect to stabilization period and learning rate of the participants.

The remainder of this paper is organized as follows: Section II describes the materials and methods used in the design and execution of the experiment. The obtained results are presented in Section III and discussed in Section IV. Finally, concluding remarks are given in Section V.

\section{Methods}

In order to analyze the effects of vibrotactile feedback patterns on human control performance in a time-critical dynamical task, a virtual inverted pendulum on a cart is both kinaesthetically and visually rendered. A wireless bracelet is used to supply additional vibrotactile feedback for the stabilization of the inverted pendulum, based on optimal control behavior of a linear quadratic regulator (LQR).

\section{A. Apparatus}

A schematic block diagram of the experimental apparatus is presented in Figure 1. It shows that the human user is only able to influence the system, i.e., stabilize the inverted pendulum, by applying force to the cart. The applied force is 


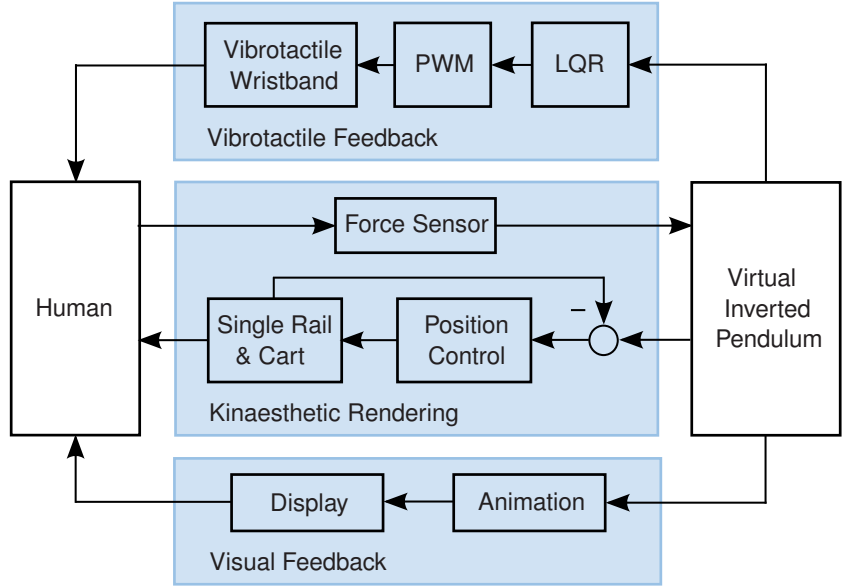

Fig. 1: Schematic block diagram of the apparatus.

measured by the force sensor and included in the simulation of the inverted pendulum. The resulting cart position is then kinaesthetically rendered. Together with the angle of the inverted pendulum, it is also used by the animation to display visual feedback to the human user. The visual feedback can be supplemented by vibrotactile feedback. Two different vibration patterns are used, representing either optimal hand velocity or acceleration profiles, both capable of stabilizing the inverted pendulum. They are calculated with respect to the optimal stabilization behavior provided by the LQR. The vibrotactile stimuli are emitted by vibratory pads situated in a wireless bracelet worn by the human user. Figure 2(a) shows a photograph of the complete experimental apparatus. Furthermore, the figure contains images of the displayed animation with full and reduced visual feedback quality.

1) Kinaesthetic rendering: The kinaesthetic rendering is actualised by a Thrusttube Module (Copley Controls, USA). It consists of a single rail stage and a linear servo motor driven cart. A vertical handle is mounted on the cart. In order to measure the applied force, a JR3-67M25 6-axis sensor (JR3 Inc., USA) is included at the base of the handle. Precise rendering of the cart position provided by the virtual inverted pendulum is ensured by PD position control.

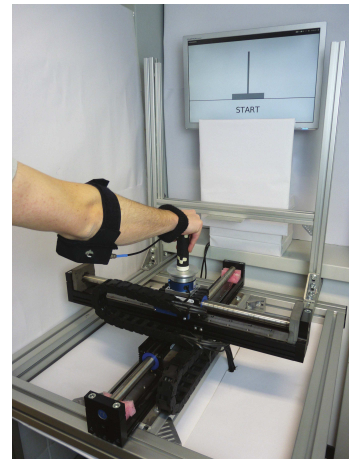

(a)

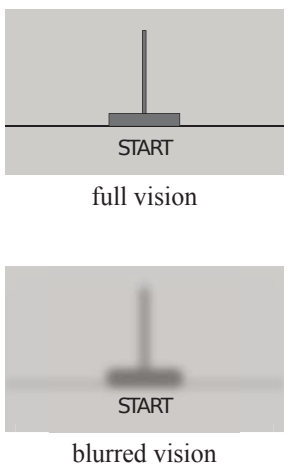

(b)
Fig. 2: a) Photograph of the apparatus b) Images of the displayed animation with full and blurred visual feedback.
In order to account for the time-critical nature of the dynamical stabilization task, attention is paid to the real-time performance of simulation and rendering. Simulink models are configured for simulation on a real-time hardware target and executed on a Linux system with real-time kernel (Ubuntu 12.04, 3.2.23, rt37) and RT-Preempt Patch. A fixed step solver is used and the sample time is set to $t_{\mathrm{s}}=1 \mathrm{~ms}$.

2) Virtual inverted pendulum: Figure 3 shows a schematic of the virtual inverted pendulum. If one defines both the cart and the pendulum to be frictionless, and the pendulum to be a uniform rod, it yields the following dynamics:

$$
\begin{aligned}
& (M+m) \ddot{x}=F-m l \dot{\theta}^{2} \sin \theta+m l \ddot{\theta} \cos \theta, \\
& \left(I+m l^{2}\right) \ddot{\theta}=m l g \sin \theta+m l \ddot{x} \cos \theta .
\end{aligned}
$$

All symbols and corresponding values are listed in Table I. The actual friction caused by the movement of the cart along the rail is negligibly small and therefore neglected. In order to make the stabilization task achievable after a short learning phase, the gravitational constant $g$ is reduced to a tenth of its real value. As previously stated, the Simulink model of the pendulum dynamics is run on real-time capable hardware.

3) Visual feedback: The current state of the pendulum is visually rendered in terms of the angle of the pendulum $\theta$ and the position of the cart $x$ within the Simulink environment. The animation is shown to the human user on a TFT display in full-screen mode. The edges of the display coincide with the movement limitations of the cart on the single rail stage. Low-contrast, greyscale colors are used for the rendering to prevent the participants from straining their eyes during the experiment. In the part of the experiment with blurred visual feedback, a translucent screen is placed directly in front of the TFT display to reduce the quality of visual feedback. Figure 2(b) shows the respective on-screen images.

4) Vibrotactile feedback: The vibrotactile wristband was developed to deliver vibrotactile stimuli to the wrist of the human user by wireless communication (as seen in Figure 2(a)). It consists of a stretchable band of fabric endowed with 4 vibratory pads, located at the center of the dorsal (top), ventral (bottom), and medial / lateral sides of the wrist. This configuration results in a minimum distance between actuators of about $4 \mathrm{~cm}$. Since this spatial separation is higher than the spatial resolution of the mechanoreceptors sensitive to high-frequency vibrations [10], it allows for reliable spatial detection by the human user. Furthermore,

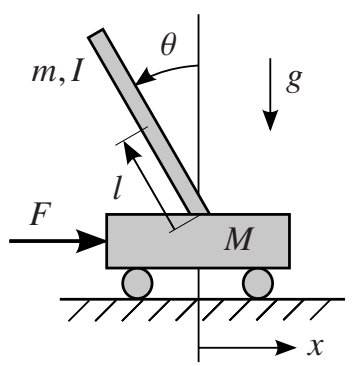

Fig. 3: Schematic of the inverted pendulum. The angle $\theta$ is controlled by the force applied by the human user $F$. 
TABLE I: Symbol explanations and values.

\begin{tabular}{|c|l|l|l|} 
Symbol & Description & Value & Unit \\
\hline$M$ & Mass of the cart & 1 & $\mathrm{~kg}$ \\
$m$ & Mass of the pendulum & 0.5 & $\mathrm{~kg}$ \\
$I$ & Inertia of the pendulum & $9.375 \cdot 10^{-4}$ & $\mathrm{~kg} \mathrm{~m}{ }^{2}$ \\
$l$ & Half length of the pendulum & 0.075 & $\mathrm{~m}$ \\
$g$ & Gravitational constant & 0.981 & $\mathrm{~m} / \mathrm{s}^{2}$ \\
$x$ & Position of the cart & & $\mathrm{m}$ \\
$\theta$ & Angle of the pendulum & & $\mathrm{deg}$ \\
$F$ & Force applied by human user & & $\mathrm{N}$
\end{tabular}

it ensures intuitiveness of spatial guidance supplied by the vibrotactile wristband by placing the stimulation sites within the same frame of reference at the anatomical landmark [11]. During the experiment, only the vibratory pads on the medial and lateral wrist joint are used, ensuring that the stimulations are consistent with the task-space coordinates and accounting for the stimulus-response compatibility [12]. Moreover, application of vibrotactile stimulation over the lateral and medial sides of the wrist guarantees that tactile sensitivities are not biased by inhomogeneous mechanoreceptor densities in hairy and glaborous skins [13].

The vibration is produced by electrical pager motors of cylindrical geometry with eccentric mass. The normal component of the resulting rotating centrifugal force is transmitted to the skin at the point of direct contact. In order to improve the transmission of the vibration to the skin, each motor is partially embedded in soft fabric and placed inside a rigid aluminium sealed capsule. The maximum frequency of actuation is $180 \mathrm{~Hz}$. This is short of the optimal response frequency of the Pacinian corpuscle, primarily responsible for perception of high-frequency vibrations $(250 \mathrm{~Hz})$ [14]. It does however still lie within its active response range [9]. The amplitude of actuation is set to a fixed value, so as not to interfere with the frequency perception [15].

Figure 4 shows the architecture of the on-board electronics of the vibrotactile wristband. The custom-designed circuit board holds a MicroController Unit (MCU), a Wireless Communication Module (WCM), a lithium-polymer battery and a USB Battery Charger (UBC). The MCU consists of a PIC18LF2431 microcontroller of the Microchip PIC family and the WCM is implemented using a $2.4 \mathrm{GHz}$ bluetooth class 1 module. The latter enables communication with the Wearable Computing Unit (WCU) by Serial Port Profile,

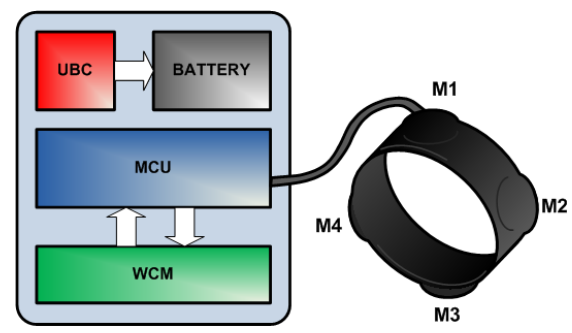

Fig. 4: Architecture of the on-board electronics composed of the MicroController Unit (MCU), the USB Battery Charger (UBC), and the Wireless Communication Module (WCM). while the UBC provides a simple and fast way to recharge the device. Measurements have shown that the vibrotactile wristband possesses a transmission delay of approx. $50 \mathrm{~ms}$. It refers to the time period that passes between the issue of a command and the occurrence of the corresponding vibration. The approximate value was determined by directly measuring the registration of the vibration at the force sensor.

\section{B. Stimuli}

The vibration patterns used for the vibrotactile feedback are based on the optimal stabilization behavior provided by the LQR [16]. In order to calculate the feedback matrix $K$, the nonlinear dynamics (1) and (2) are first linearized about the vertically upward equilibrium position at angle $\theta=0$. This yields the state-space representation

$$
\begin{aligned}
& {\left[\begin{array}{c}
\dot{x} \\
\ddot{x} \\
\dot{\theta} \\
\ddot{\theta}
\end{array}\right]=\left[\begin{array}{cccc}
0 & 1 & 0 & 0 \\
0 & 0 & \frac{m^{2} l^{2} g}{v} & 0 \\
0 & 0 & 0 & 1 \\
0 & 0 & \frac{m(M+m) l g}{v} & 0
\end{array}\right]\left[\begin{array}{c}
x \\
\dot{x} \\
\theta \\
\dot{\theta}
\end{array}\right]+\left[\begin{array}{c}
0 \\
\frac{m l^{2}+I}{v} \\
0 \\
\frac{m l}{v}
\end{array}\right] F} \\
& \text { with } v=M m l^{2}+I(M+m)
\end{aligned}
$$

and the following open-loop eigenvalues:

$$
\lambda_{o l, 1 / 2}=0, \quad \lambda_{o l, 3 / 4}= \pm 2.5573 .
$$

The corresponding eigenvectors show that $\lambda_{o l, 1 / 2}$ exclusively correspond to the position of the cart $x$, while $\lambda_{o l, 3 / 4}$ are strongly influenced by the angle $\theta$ and its derivative $\dot{\theta}$. The latter affirms the time-critical, unstable nature of the dynamics of the virtual inverted pendulum system.

The elements of the weighting matrices $Q$ and $R$ used to determine the feedback matrix $K$ are chosen so that the resulting LQR prioritizes the regulation of the angular deviation from the vertically upward equilibrium position:

$$
Q=\left[\begin{array}{cccc}
10^{3} & 0 & 0 & 0 \\
0 & 1 & 0 & 0 \\
0 & 0 & 10^{5} & 0 \\
0 & 0 & 0 & 10^{3}
\end{array}\right], \quad R=1
$$

The resulting LQR feedback matrix reads as follows:

$$
K=\left[\begin{array}{llll}
-31.62 & -147.39 & 338.40 & 59.70
\end{array}\right] \text {. }
$$

If one applies this matrix and the state vector $\underline{x}=[x, \dot{x}, \theta, \dot{\theta}]^{T}$, as previously defined in (3), in the state feedback law

$$
F=-K \underline{x},
$$

it results in the following closed-loop eigenvalues $\lambda_{c l, i}$ and the corresponding time constants $T_{i}=-\operatorname{Re}\left\{\lambda_{i}\right\}^{-1}$ :

$$
\begin{aligned}
\lambda_{c l, 1} & =-140.24, & T_{1} & =0.0071 \mathrm{sec}, \\
\lambda_{c l, 2} & =-10.02, & T_{2} & =0.0998 \mathrm{sec}, \\
\lambda_{c l, 3 / 4} & =-0.22 \pm i 0.22, & T_{3 / 4} & =4.4775 \mathrm{sec} .
\end{aligned}
$$

When looking at the respective eigenvectors, one sees that the slow eigenvalues $\lambda_{c l, 3 / 4}$ are mainly influenced by the position of the cart $x$ and the fast eigenvalues $\lambda_{c l, 1 / 2}$ mostly correspond to the angular velocity $\dot{\theta}$. This is consistent with the configuration of the LQR weighting matrices in (5). 
The information about the LQR reference behavior is supplied by means of two different discrete vibration patterns (acceleration-profile and velocity-profile based feedback). Both patterns are implemented as a form of pulse width modulation, in which the pulse duration $t_{\text {on }}$ possesses a constant value of $50 \mathrm{~ms}$ and the time between pulses $t_{\text {off }}$ as well as the stimulation site on the wrist are defined by the LQR signal. Before the vibration pattern is calculated, a temporal extrapolation with $\Delta t=200 \mathrm{~ms}$ is performed to compensate for response delay in sensory-motor control of the human user ( $150 \mathrm{~ms},[17],[18])$ as well as transmission delay of the vibrotactile wristband ( $\sim 50 \mathrm{~ms})$.

1) Acceleration-profile based feedback: For this feedback, the temporal extrapolation is based on the current states of the system $\underline{x}(t)$ as well as the force currently applied by the human user $F_{\mathrm{h}}(t)$. Both values are inserted into (3), to obtain the derivatives of the current states of the system:

$$
\underline{\dot{x}}(t)=f\left(\underline{x}(t), F_{\mathrm{h}}(t)\right) .
$$

Subsequently, the temporal extrapolation is performed:

$$
\begin{aligned}
& x(t+\Delta t)=\frac{1}{2} \ddot{x}(t) \Delta t^{2}+x(t), \\
& \dot{x}(t+\Delta t)=\ddot{x}(t) \Delta t+\dot{x}(t), \\
& \theta(t+\Delta t)=\frac{1}{2} \ddot{\theta}(t) \Delta t^{2}+\theta(t), \\
& \dot{\theta}(t+\Delta t)=\ddot{\theta}(t) \Delta t+\dot{\theta}(t) .
\end{aligned}
$$

The predicted states of the system $\underline{x}(t+\Delta t)$ are then used in a state feedback law to obtain a hypothetical LQR force

$$
F_{\text {hyp }}=F_{\text {lqr }}(t+\Delta t)=-K \underline{x}(t+\Delta t) .
$$

It represents the reference behavior, which is used for the mapping of the vibration pattern. The side of the vibration is defined by the sign of $F_{\text {hyp }}$; a negative, left-facing force leads to a vibration on the left and a positive, right-facing force leads to a vibration on the right side of the wrist.

The time between pulses $t_{\text {off }}$ is defined by the intensity of the hypothetical LQR force $F_{\text {hyp }}$. To ensure that the value of $F_{\text {hyp }}$ lies within a fixed interval, it's absolute value $\left|F_{\text {hyp }}\right|$ is first saturated at a previously determined threshold $F_{\text {sat }}$. Subsequently, a normalized exponential function is used to map different intensity levels of $\left|F_{\text {hyp }}\right| \in\left[0, F_{\text {sat }}\right]$ to a total of 10 different values for $t_{\mathrm{off}}-$ starting at $t_{\mathrm{off}, \min }=100 \mathrm{~ms}$ and leading up to $t_{\mathrm{off}, \max }=1 \mathrm{~s}$ (Figure 5). Thus, the highest force intensity level correlates to the highest pulse frequency, i.e., the shortest time between pulses and vice versa.

2) Velocity-profile based feedback: Here, instead of the hypothetical LQR force $F_{\text {hyp }}$, the hypothetical velocity $\dot{x}_{\text {hyp }}$ represents the reference behavior used for the mapping of the vibration pattern. Thus, instead of the force currently applied by the human user $F_{\mathrm{h}}(t)$, the force that would currently be applied by the LQR $F_{\mathrm{lqr}}(t)$ is inserted into (3) to calculate the derivatives of the current states of the system:

$$
\underline{\dot{x}}(t)=f\left(\underline{x}(t), F_{\mathrm{lqr}}(t)\right)=f(\underline{x}(t),-K \underline{x}(t)) .
$$

Subsequently, the same temporal extrapolation as in (11) is used to obtain the hypothetical velocity $\dot{x}_{\text {hyp }}=\dot{x}(t+\Delta t)$.
Apart from these differences, the vibration pattern design is performed analogously to the acceleration-profile case. The vibrotactile stimulation site depends on the sign of $\dot{x}_{\text {hyp }}$ and the time between pulses $t_{\text {off }}$ is defined by its intensity.

\section{Design $\mathcal{E}$ Procedure}

1) Design: The present study was conducted in a $3 \times 2$ mixed design. The between-groups factor was given by the type of vibrotactile feedback. The participants were randomly assigned to perform the experiment with either velocity-profile based, acceleration-profile based, or without vibrotactile feedback. The within-subject factor was the level of visual feedback, encompassing (in order of appearance) full- or blurred-vision. During the first phase, the participants performed the task under full-vision. Subsequently, in the blurred-vision phase, the participants performed the task with the translucent screen positioned in front of the TFT display. Each phase lasted for approximately 30 minutes.

2) Procedure: The participants were told to stand in front of the apparatus, directly facing the screen. Their standing was adjusted, so that they could comfortably hold the manipulandum with their dominant hand. The participants placed their palm on top of the manipulandum and powergripped it, in order to align the direction of the vibrotactile stimulation with the lateral movement of the manipulandum along the single rail stage. They were instructed to keep the inverted pendulum vertically stabilized as long as possible. A trial ended when the absolute angular deviation of the pendulum from the vertically upright equilibrium position surpassed $45^{\circ}$ or when the animated cart reached either edge of the animation. The participants, who were supplied with additional vibrotactile feedback wore the vibrotactile bracelet on the wrist of the dominant arm.

Prior to the experiment, the manipulandum was positioned at the center of the apparatus. At the beginning of each trial, a 5 second countdown was numerically displayed on screen below the animation. During the countdown, the position of the manipulandum was fixed and the inverted pendulum was locked in the vertically upright equilibrium position. When the countdown was over, the animated pendulum changed its color, signalling the beginning of the trial and the participant could move the manipulandum at will. Due to slight noise in the measurement of the force sensor, the pendulum naturally fell to either direction at the beginning of the trial. When the trial ended, the manipulandum stopped moving and the

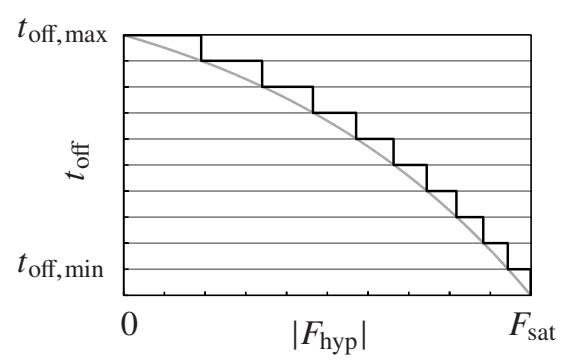

Fig. 5: Exponential function used to assign intensity levels of the hypothetical force $F_{\text {hyp }}$ to the time intervals $t_{\text {off }}$. 
animated pendulum changed its color back to the original. The manipulandum then moved back to the center of the apparatus, preparing for the start of the following trail.

3) Participants: A total of 30 participants volunteered to take part in this study. All participants were right handed and had normal or corrected-to-normal vision. The average age of the participants was 27.0 years at the time of testing, and 4 of them were female participants. Informed consent was obtained from all the participants before they took part in the study. The research ethics was obtained from the ethics committee at Technische Universität München.

\section{Results}

We characterized the performance of the participants in terms of stabilization period and corresponding learning rate. The performance change over the course of the experiment was approximated by an exponential function.

$$
y(k)=a \cdot e^{b k}
$$

where $k$ is the trial number, $a$ is a scaling factor and $b$ is the growth rate. Figure 6 shows the exemplary data of a single participant, including the fitted exponential function. Figure 7 shows the results of the statistical analysis.

The analysis of the overall stabilization period showed that, with full visual feedback, the participants with the velocity-dependent feedback were able to keep the inverted pendulum stabilized for the longest time $(28.2 \pm 36.0 \mathrm{sec})$. The stabilization period was markedly shorter without any vibrotactile feedback $(9.7 \pm 6.6 \mathrm{sec})$ and it was shortest with the acceleration-dependent feedback $(8.6 \pm 3.2 \mathrm{sec})$. When blurring was introduced to the visual feedback, the stabilization period was generally much shorter. Nonetheless, a small advantage of the velocity-dependent vibrotactile feedback $(8.2 \pm 2.7 \mathrm{sec})$ was observed, in comparison to acceleration-dependent $(6.1 \pm 1.2 \mathrm{sec})$ and no feedback $(5.3 \pm 1.3 \mathrm{sec})$. Due to the large individual performance differences inbetween the participants supplied with velocitydependent feedback, a mixed $3 \times 2$ did not indicate the main effect of vibrotactile feedback $(p=0.06)$. However, reduction of stabilization period due to the blurred vision was statistically supported, $\mathrm{F}(1,27)=5.864, p=0.02$. The interaction effect was not significant $(p=0.12)$.

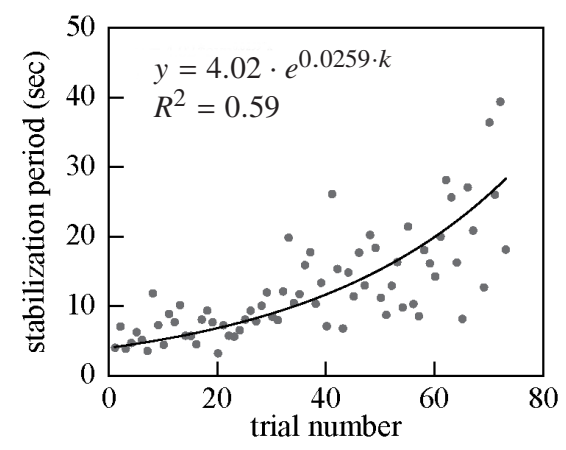

Fig. 6: Stabilization period over trial number. Data of a single participant and the fitted exponential function are shown.
The learning rate in terms of the growth rate $b$ in (16) is shown in Figure 7. The descriptive analysis indicated that, with full visual feedback, the learning rate was highest with the velocity-dependent feedback $(b=0.0605)$, followed by no vibrotactile feedback (0.0174) and it was slowest with the acceleration-dependent feedback (0.0155). The learning rates with blurred vision were as follows; velocity-dependent (0.0078), acceleration-dependent (0.0061), and no feedback (0.0023). As the growth rates were not normally distributed, a Kruskal-Wallis test was used to statistically compare the performance differences between the three vibrotactile conditions for each visual feedback type. The test revealed a significant effect of vibrotactile feedback during the full vision phase $\left(\chi^{2}(2)=7.70, p=0.021\right)$. A post-hoc test using Mann-Whitney tests supported increased growth rate with the velocity-dependent feedback in comparison with the acceleration-dependent feedback $(p=0.005)$ and no vibrotactile feedback $(p=0.016)$. In contrast, during the blurred vision phase, no significant effect of additional vibrotactile feedback on performance was observed $(p=0.515)$.

\section{Discussion}

The analysis of the experimental phase with full visual feedback showed that the velocity-dependent vibrotactile feedback leads to a significant improvement in dynamic object stabilization performance. In contrast, accelerationdependent feedback resulted in slightly inferior stabilization performance, compared to no vibrotactile feedback.

The results also showed that the velocity-dependent feedback induced the highest learning rate, further supporting that the extraneous feedback leads to an overall improvement of human motor control. Although the negative effect of acceleration-dependent feedback was not expected, the improved performance with the velocity-dependent feedback is consistent with our hypothesis that velocity information about the arm dynamics is more directly transformed into motor commands in the central nervous system.

When the quality of the visual feedback is degraded with blurred vision, differences in stabilization times and learning rates between the three feedback types are significantly reduced. Although a slight advantage of the participants with
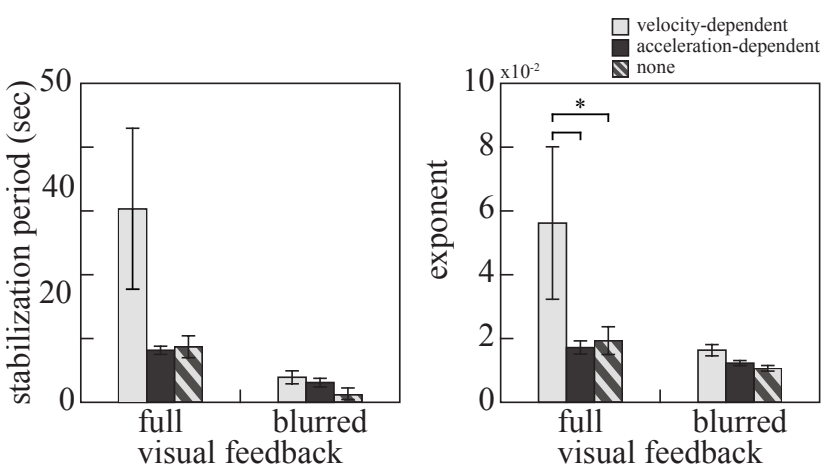

Fig. 7: Stabilization period and learning rate represented by the exponential coefficient averaged across participants. Error bars represent one standard deviation. $* p=0.05$ 
vibrotactile feedbacks is observable, vibrotactile feedback is not sufficient in singularly guiding time-critical behavior when the quality of visual feedback is significantly reduced. Thus, it seems that the haptic feedback assists visuo-motor coordination by priming an appropriate response rather than explicitly guiding the behavior by itself - at least during highly dynamic motor coordination.

When used in combination with full visual feedback, the effectiveness of vibrotactile feedback depends strongly on its vibration pattern. While velocity-dependent feedback improved overall dynamic object stabilization performance, acceleration-dependent feedback even slightly reduced it by inappropriately priming visuo-motor coordination. This is consistent with our exit interview, in which several of the participants stated that the acceleration-dependent feedback distracted them from focusing on the visual feedback.

The effect of vibrotactile feedback design may explain the markedly different results from those presented in [7]. Directional cueing by vibrotactile feedback is reported to be effective for spatial guidance, when the respective target is either stable or possesses slow dynamics [2], [19], [20]. However, our stabilization task is inherently unstable and was specifically designed for time-critical dynamics. We believe that in our study the vibrotactile feedback was influencing low-level control by priming visuo-motor coupling, which led to the selectiveness of the feedback design for improving the overall human control performance. Though the precise dynamics of the inverted pendulum in [7] are unreported, one may assume that their task was less time-critical, given the fact that their participants were able to perform the task, i.e., stabilize the pendulum, much longer from the beginning of the experiment on. With such slow dynamics, vibrotactile feedback may be processed for high-level motor planning and can be designed flexibly in expense of response delay.

\section{Conclusion}

In summary, this study reported how a human-centric design of vibrotactile feedback can improve sensory-motor coordination of human participants in a time-critical object stabilization task. However, this holds true only, when the vibrotactile feedback is supplement to an additional source of feedback, e.g., visual feedback. When precise time-critical, spatio-temporal motor coordination is required, vibrotactile feedback may bias low-level visuo-motor mapping. Thus, optimal design of the vibrotactile feedback is most critical, in order to ensure appropriate priming of user responses.

\section{AcKNOWLEDGMENT}

The research leading to these results has received funding from the European Union Seventh Framework Programme FP7/2007-2013 under grant agreement $n^{\circ} 601165$ of project "WEARHAP - WEARable HAPtics for humans and robots". The authors would like to thank Claudio Loconsole and Edoardo Sotgiu for their contributions to the development of the vibrotactile wristband and Paul Färber for his assistance in the implementation of the experimental apparatus.

\section{REFERENCES}

[1] L. R. Elliott, M. Coovert, M. Prewett, A. Walvord, K. Saboe, and R. Johnson, A Review and Meta Analysis of Vibrotactile and Visual Information Displays. U.S. Army Research Laboratory Aberdeen Proving Ground, MD, 2009.

[2] J. B. F. V. Erp, H. A. H. C. V. Veen, C. Jansen, and T. Dobbins, "Waypoint navigation with a vibrotactile waist belt," ACM Trans. Appl. Percept., vol. 2, no. 2, pp. 106-117, Apr. 2005.

[3] K. Bark, P. Khanna, R. Irwin, P. Kapur, S. A. Jax, L. J. Buxbaum, and K. J. Kuchenbecker, "Lessons in using vibrotactile feedback to guide fast arm motions," in 2011 IEEE World Haptics Conference. IEEE, Jun. 2011, pp. 355-360.

[4] E. Ruffaldi, A. Filippeschi, A. Frisoli, O. Sandoval, C. A. Avizzano, and M. Bergamasco, "Vibrotactile perception assessment for a rowing training system," in World Haptics 2009 - Third Joint EuroHaptics conference and Symposium on Haptic Interfaces for Virtual Environment and Teleoperator Systems. IEEE, 2009, pp. 350-355.

[5] L. Jones and N. Sarter, "Tactile Displays: Guidance for Their Design and Application," Human Factors: The Journal of the Human Factors and Ergonomics Society, vol. 50, no. 1, pp. 90-111, Feb. 2008.

[6] S. Choi and K. J. Kuchenbecker, "Vibrotactile Display: Perception, Technology, and Applications," Proceedings of the IEEE, vol. 101, no. 9, pp. 2093-2104, Sep. 2013.

[7] E. Tzorakoleftherakis, F. Mussa-Ivaldi, R. Scheidt, and T. Murphey, "Effects of optimal tactile feedback in balancing tasks: A pilot study," in American Control Conference (ACC), June 2014, pp. 778-783.

[8] E. J. Hwang, M. A. Smith, and R. Shadmehr, "Adaptation and generalization in acceleration dependent force fields," Exp Brain Res, vol. 169, no. 4, pp. 496-506, Mar. 2006.

[9] J. Bell, S. Bolanowski, and M. H. Holmes, "The structure and function of pacinian corpuscles: A review," Progress in Neurobiology, vol. 42, no. 1, pp. 79-128, Jan. 1994.

[10] F. Gemperle, T. Hirsch, A. Goode, J. Pearce, D. Siewiorek, and A. Smailigic, "Wearable vibro-tactile display," Carnegie Mellon Wearable Group, Carnegie Mellon University, Tech. Rep., 2003.

[11] R. W. Cholewiak and A. A. Collins, "Vibrotactile localization on the arm: Effects of place, space, and age," Perception $\mathcal{E}$ Psychophysics, vol. 65 , no. 7, pp. 1058-1077, Oct. 2003.

[12] C. Jansen, A. Oving, and H. V. Veen, "Vibrotactile movement initiation," Proceedings of Eurohaptics, pp. 110-117, 2004.

[13] D. A. Mahns, N. M. Perkins, V. Sahai, L. Robinson, and M. J. Rowe, "Vibrotactile frequency discrimination in human hairy skin." Journal of neurophysiology, vol. 95, no. 3, pp. 1442-50, Mar. 2006.

[14] M.-J. Yoon and K.-H. Yu, "Psychophysical experiment of vibrotactile pattern perception by human fingertip," Neural Systems and Rehabilitation Engineering, IEEE Transactions on, vol. 16, no. 2, pp. 171-177, April 2008.

[15] J. Morley and M. Rowe, "Perceived pitch of vibrotactile stimuli: effects of vibration amplitude, and implications for vibration frequency coding," The Journal of physiology, vol. 431, pp. 403-16, Dec. 1990.

[16] F. L. Lewis, D. Vrabie, and V. L. Syrmos, Optimal control. John Wiley \& Sons, 2012.

[17] L. García-Larrea, A. C. Lukaszewicz, and F. Mauguière, "Somatosensory responses during selective spatial attention: The N120-to-N140 transition." Psychophysiology, vol. 32, no. 6, pp. 526-37, Nov. 1995.

[18] R. Zopf, C. M. Giabbiconi, T. Gruber, and M. M. Müller, "Attentional modulation of the human somatosensory evoked potential in a trialby-trial spatial cueing and sustained spatial attention task measured with high density 128 channels EEG." Brain research. Cognitive brain research, vol. 20, pp. 491-509, 2004.

[19] A. Cosgun, E. Sisbot, and H. Christensen, "Guidance for human navigation using a vibro-tactile belt interface and robot-like motion planning," in Robotics and Automation (ICRA), IEEE International Conference on, May 2014, pp. 6350-6355.

[20] V. Lehtinen, A. Oulasvirta, A. Salovaara, and P. Nurmi, "Dynamic tactile guidance for visual search tasks," in Proceedings of the 25th Annual ACM Symposium on User Interface Software and Technology, ser. UIST '12. New York, NY, USA: ACM, 2012, pp. 445-452. 